\title{
Kuttner's tumor of bilateral submandibular and parotid glands
}

\begin{abstract}
Chronic sclerosing sialadenitis, known as Kuttner tumor (KT), is a chronic inflammatory disease of the salivary glands. KT is a benign condition but presents with a hard mass that clinically mimicks salivary gland neoplasms. It is an underdiagnosed entity among pathologist and otolaryngologist even tough it was first reported by Kuttner more than a century ago. It might be due to rare occurence and not to have malign histologic features. KT primarily affects one of the submandibular glands. Herein, we report a case of KT presenting with time-serial involvement of the bilateral submandibular and parotid glands. This case would be of particular interest to head and neck surgeons and pathologists, in order to create more awareness with regard to KT which is a not well-known entity.
\end{abstract}

Keywords: salivary gland, submandibular gland, kuttner tumor, chronic sclerosing sialadenitis
Volume 2 Issue 2 - 2015

\author{
Umit Aydin,' Omer Karakoc,' Fatih Arslan,' \\ Ali Fuat Cicek, ${ }^{2}$ Omer Gunhan ${ }^{2}$ \\ 'Gulhane Military Medical Academy, Department of \\ Otolaryngology, Turkey \\ ${ }^{2}$ Gulhane Military Medical Academy, Department of Pathology, \\ Turkey
}

Correspondence: Umit Aydin, Gulhane Military Medical Academy, Department of Otolaryngology, 060 I8, Etlik, Ankara,

Turkey,Tel+903 | 23045707, Email denizsea30@gmail.com

Received: January 14, 2015 | Published: February 2, 2015
Abbreviations: KT, kuttner tumor; ANA, anti-nuclear antibody; IgG4, 1mmunoglobulin g4

\section{Introduction}

Chronic sclerosing sialadenitis, known as KT, was first described in 1896 by Kuttner. KT is clinically characterised by a firm mass of submandibular gland in middle-aged adults. This is an uncommon tumor-like lesion that typically involves one of the submandibular glands. ${ }^{1-3}$ The treatment of choice is complete removal of the affected gland. The definitive diagnosis is made by histopathological examination. Histologically, KT is characterized by lymphoid follicle, acinar atropy, periductal fibrosis and dilated ducts. it is a benign and relatively uncommon condition. Therefore, it may be a little difficult for a pathologists to distinguish KT from other forms of sialadenitis. Multiglandular involvement of KT rarely occurs and it might be a manifestation of IgG4-related systemic sclerosing disease., ${ }^{4,5} \mathrm{We}$ experienced a rare case of KT that presents with a time-serial involvement of the bilateral submandibular and parotid glands. This report described the histological, clinical features and management of this patient. We aimed to raise awareness among otolaryngologists and pathologists about a not well-known and under-recognized entity.

\section{Case report}

A 60-year-old woman was admitted to our clinic with the complaint of a slow-growing mass in the left submandibular region for 4 months. She had no history of drying eyes or mouth. She was with normal general health and no other personal history of disease. Physical examination revealed a very firm, slightly tender, $4 \times 3 \mathrm{~cm}$ sized left submandibular mass. Ultrasonography showed diffuse enlargement with impaired parenchymal structure in the left submandibular gland. But there was no sign of sialolithiasis. No other masses or adenopathy were noted in the head and neck in physical examination and ultrasonography. Flexible endoscopic examination of the nasopharynx, hypopharynx, larynx was normal. Fine needle aspiration cytology showed a non-specific inflammation. Routine laboratory tests including full blood count, white cell count, liver function tests, erythrocite sedimentation rate were within normal range. Left submandibular gland excision was performed under general anesthesia. Light microscopic examination of tissue stained with hematoxylin and eosin revealed remarkable fibrosis, accompanied by the inflammatory infiltrate and lymphoid follicules (Figure 1A). Acinar atrophy, concentric periductal sclerosis("onionskin" appearance), and ductal ectasy were also observed (Figure 1b). But there were no neoplastic features. All these findings were consistent with chronic sclerosan sialadenitis. The patient recovered well after operation. But she presented with a prominent right submandibular mass and mild bilateral symmetric parotid swelling three months later (Figures 2A \& 2B). Right submandibular mass had the same clinical features of the left. Computed tomography of the neck with contrast was also performed. It showed diffuse enlargement of the right submandibular and bilateral parotid gland which enhance moderately (Figure 3). The patient underwent operation again and right submandibular gland was surgically removed. Histological features of the excised right submandibular gland same as the left submandibular gland. Specimens of the bilateral submandibular glands displayed the histopathologic features of the chronic sclerosing sialadenitis. Bilateral submandibular gland specimens predominantly showed the stage $2 \mathrm{KT}$ according to Seifert's histopathologic classification (Table 1). We also performed immunohistochemical staining. Inflammatory infiltrate were composed of CD4+ T cells (helper T cell) and CD8+ T cells (cytotoxic $\mathrm{T}$ lymphocyte) in periductal area (Figures 4A\&4B). A large number of $C D 138+$ cells(plasma cell) were also observed in interfolliculer area (Figure 4c). Lymphoid follicules predominantly consisted CD 20+ cells (B lymphocyte) in the germinal center (Figure 4D). Patient was also positive for anti-nuclear antibody(ANA) in blood. These findings indicated that it may be an immune-mediated process and the patient was referred to an immunologist for excluding the autoimmune diseases. Other autoimmun antibodies were not detected. IgG and IgG4 level that might be elevated in IgG4-related systemic sclerosing disease were also normal range. Immunologist reported to have no evidence of otoimmun diseases in the patient because there was no sign of autoimmun disorder except the positive antinuclear antibody that considered as non-spesific. The patient was followed for 6 months without further therapy. No other neck masses detected in this period except bilateral mild parotid swelling. Patient did not mentioned dry mouth or any other symptom after surgery. 

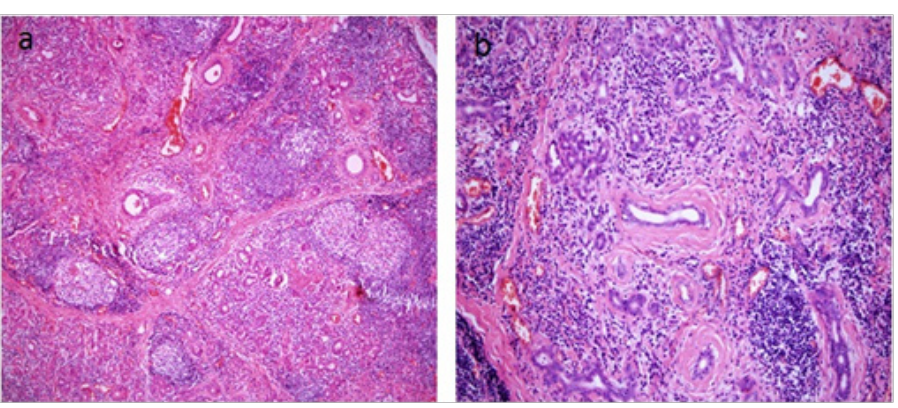

Figure I (a) Remarkable fibrosis, inflammatory infiltrate and lymphoid follicules, $\times 40 \mathrm{H} \& \mathrm{E}$ (b) Acinar atrophy, ductal ectasy and concentric periductal sclerosis, $x 100 \mathrm{H} \& \mathrm{E}$.

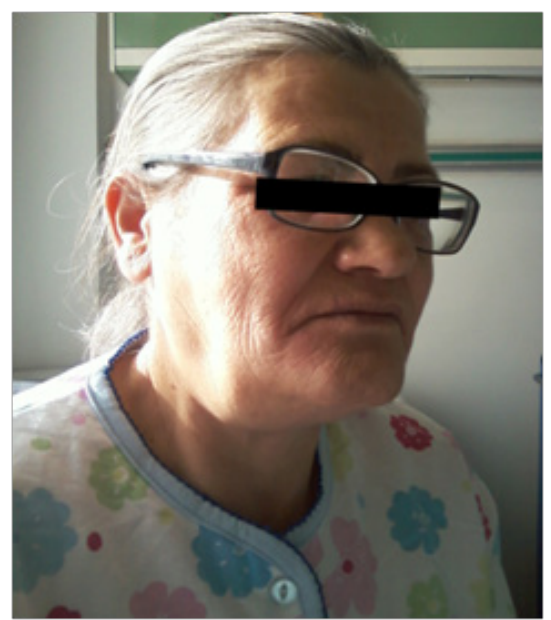

Figure 2 Prominent right submandibular mass.
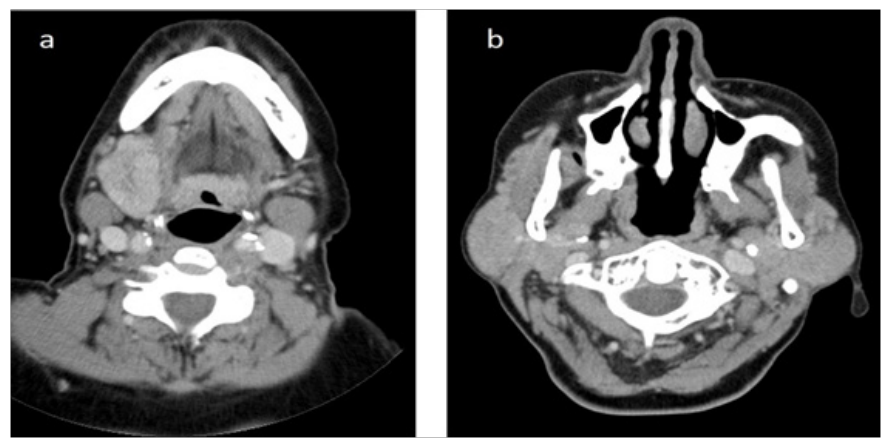

Figure 3 Axial CT scan showing the enlargement of the right submandibular gland (a) and bilateral parotid glands (b).

\section{Discussion}

$\mathrm{KT}$ is an chronic inflammatory process that involves the salivary glands. It is a rare entity and typically affects one of the submandibular glands. KT presents with a slow-growing, hard mass in the submandibular region and clinically mimics a neoplasm of salivary gland. This disease is classified as a tumor-like lesion of the salivary glands by the World Health Organization. ${ }^{6}$ The diagnosis of $\mathrm{KT}$ is based on histologic findings. Acinar loss, dense fibrosis, ductal dilatation and lymphoid follicle formation are the characteristic histologic features.

Several hypotheses have been suggested to explain the origin of the KT. Sialolithiasis were demonstrated in $29 \%-83 \%$ of cases. Some authors state that stones cause the obstruction of salivary flow. Stasis of secretions induce the inflammation and fibrosis which resulted with chronic sclerosing sialadenitis. Whereas, others state that the formation of calculi is secondary to the sialadenitis. Seifert and Donath have proposed a theory of obstructive electrolyte sialadenitis. ${ }^{7}$ This hypothesis claims that a secretion disorder produce mucus plugs obstructing the small ducts and also cause changes in the electrolyte content which leads to immune process. Neverthless, etiology is still unclear.
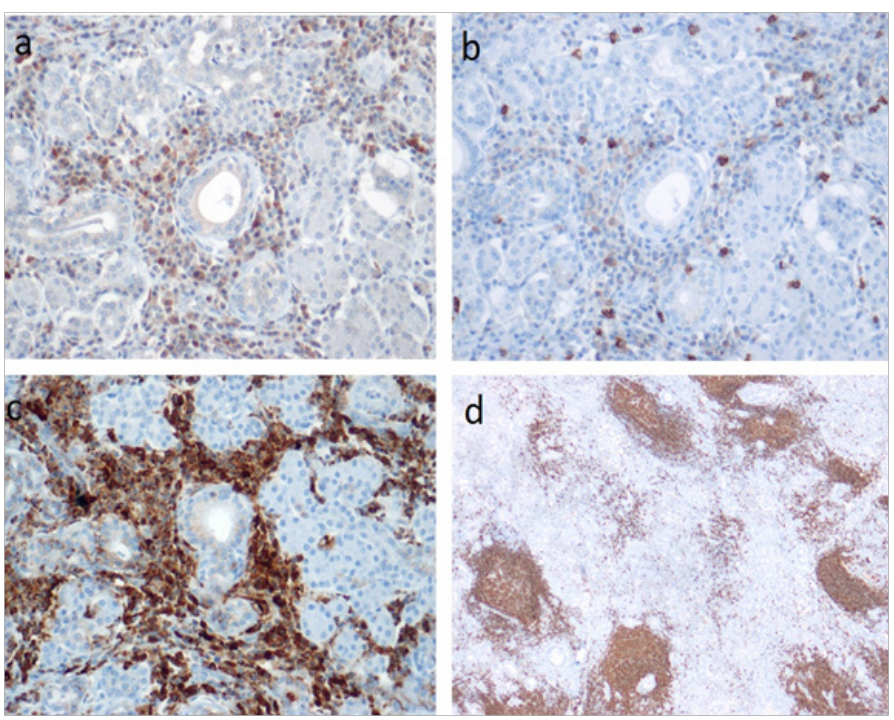

Figure 4 (a) Prominent periductal CD4+ lymphocytic infiltration, x200 CD4 immunostaining (b) Mild periductal CD8 + lymphocytic infiltration, x200 CD8 immunostainining (c) Dense plasma cell infiltration in stroma, $\times 200$ CDI38 immunostaining (d) B lymphocytes in the lymph follicules, x40 CD20 immunostaining.

A histological diagnosis is necessary because KT usually present with the proggresive enlargement of the submandibular gland that mimicking a true neoplasm. Histological features of KT are marked fibrosis, inflammatory infiltration, lymphoid follicles, loss of acini, periductal fibrosis and dilated ducts. Lymphocytes and plasma cells are predominant in the inflammatory infiltrate. Dilated ducts usually contain inspissated secretion. Fibrosis begins in the periductal region in an onion-skin arrangement and extends into the interlobular septa. According to Seifert, ${ }^{7}$ severity of inflammation in KT may progress through 4 different histological stages (Table 1).

Multiple involvement of salivary glands is an extremely rare condition and suggests that $\mathrm{KT}$ is closely related to an immune process. ${ }^{5}$ In literature, it is reported that KT may be related with the Immunoglobulin G4(IgG4)-related systemic sclerosing disease which is a chronic autoimmune disorder with diverse clinical presentation such as retroperitoneal fibrosis, sclerosing cholangitis, and sclerosing pancreatitis. Therefore, we performed investigations for the autoimmun disease. Immunocytochemical investigations on sections from the glands revealed predominatly $\mathrm{CD} 4+$ lymphocytes and plasma cells in the inflamatory infiltrate. There was a preponderance of CD4 over CD8 lymphocytes. Prominent B-cell reaction was noticed in the lymph follicles. These findings suggested an sialadenitis related with immun process. Autoimmun antibody except the ANA were negative. IgG and IG4 levels were in normal range. Patient was referred to an 
immunologist with these findings. But no otoimmun disorder reported by the immunologist.

Table I Seifert's histological staging of KT

\begin{tabular}{|c|c|}
\hline Stage I & $\begin{array}{l}\text { Focal chronic inflammation with accumulation of } \\
\text { lymphocytes around moderately dilated salivary ducts that } \\
\text { contain inspissated secretion }\end{array}$ \\
\hline Stage 2 & $\begin{array}{l}\text { Severe periductal fibrosis and diffuse lymphocytic infiltration } \\
\text { are more noticeable. The ductal system shows inspissated } \\
\text { secretion and focal metaplasia with proliferation of ductal } \\
\text { epithelium. Periductal lymphoid follicles are well developed. } \\
\text { There is fibrosis in the centres of the lobules along with the } \\
\text { atrophy of acini. }\end{array}$ \\
\hline Stage 3 & $\begin{array}{l}\text { Even more prominent lymphocytic infiltration with lymphoid } \\
\text { follicle formation, parenchymal atrophy, periductal sclerosis } \\
\text { in addition to squamous and goblet cell metaplasia in the } \\
\text { ductal system. }\end{array}$ \\
\hline Stag & $\begin{array}{l}\text { Diffuse sclerosis and prominent parenchymal loss cause } \\
\text { cirrhosis-like appearance (end-stage, the "burnt-out"phase). }\end{array}$ \\
\hline
\end{tabular}

Possible differential diagnoses of KT include other benign lesions of salivary glands such as Kimura's disease, Sjogren's syndrome, simple chronic sialadenitis, granulomatous sialadenitis. $\mathrm{KT}$ is differantiated from others with prominent sclerosis and lack of epimyoepithelial islands.

Treatment of the KT is the removal of the affected salivary gland Hovewer, management can be conservative if the mass is asymptomatic and there is enough evidence that the lesion is benign. Cheuk et al suggested that sonography and sonographically guided needle biopsy may indicate for KT and prevent operative excision of an otherwise benign condition. ${ }^{8}$ Hovewer, it is usually difficult to differentiate KT from a true neoplasm preoperatively. A hard mass in the submandibular gland is highly suggestive of tumor. Therefore, the submandibular gland is most commonly surgically removed.

Kuttner's tumor is a rare benign disease that mimics true neoplasm due to presentation with unilateral firm submandibular mass. Final diagnosis of $\mathrm{KT}$ is established by the histopathological examination.
KT is generally treated with surgical removal of the affected salivary gland and no further treatment is required. However, in some cases, KT may be a manifestation of a systemic IgG4-related autoimmune disease. Therefore, patients diagnosed with KT should be referred to a immunologist to exclude an autoimmune disorder. Both surgeon and pathologist KT must be aware of this benign condition for the correct diagnosis of $\mathrm{KT}$.

\section{Acknowledgments}

None.

\section{Conflicts of interest}

The authors declare that there are no conflicts of interest.

\section{References}

1. Unal M, Karabacak T. Kuttner's tumor of the submandibular gland. B-ENT. 2006;2(4):197-199.

2. Adachi M, Fujita Y, Murata T, et al. A case of Kuttner tumor of the submandibular gland. Auris Nasus Larynx. 2004;31(3):309-312.

3. Kiverniti E, Singh A, Clarke P. Kuttner's tumor: an unusual cause of salivary gland enlargement. Hippokratia. 2008;12(1):56-58.

4. Roh JL, Kim JM. Kuttner's tumor: unusual presentation with bilateral involvement of the lacrimal and submandibular glands. Acta Otolaryngol. 2005;125(7):792-796.

5. Shin YU, Oh YH, Lee YJ. Unusual Involvement of IgG4-Related Sclerosing Disease in Lacrimal and Submandibular Glands and Extraocular Muscles. Korean J Ophthalmol. 2012;26(3):216-221.

6. Seifert G. Tumor-like lesions of the salivary glands. The new WHO classification. Pathol Res Pract. 1992;188(7):836-846.

7. Seifert G, Donath K. On the pathogenesis of the Küttner tumor of the submandibular gland -- Analysis of 349 cases with chronic sialadenitis of the submandibular (author's transl). HNO. 1977;25(3):81-92.

8. Cheuk W, Chan JK. Kuttner Tumor of the Submandibular Gland FineNeedle Aspiration Cytologic Findings of Seven Cases. Am J Clin Pathol. 2002;117(1):103-108. 